INTERNATIONAL RESEARCH JOURNAL OF PHARMACY

www.irjponline.com

ISSN $2230-8407$

Review Article

\title{
ENHANCEMENT OF BIOAVAILABILITY THROUGH INCREASE IN DRUG PERMEATION, STABILITY AND RETENTION TIME
}

\author{
Vishal Sachdeva*, Mahesh Kumar Kataria \\ Seth G. L. Bihani S. D. College of Technical Education, Sri Ganganagar, Rajasthan, India \\ *Corresponding Author Email: sachdeva.pharmacy@gmail.com
}

Article Received on: 16/03/13 Revised on: 09/04/13 Approved for publication: 01/05/13

DOI: 10.7897/2230-8407.04513

IRJP is an official publication of Moksha Publishing House. Website: www.mokshaph.com

(C) All rights reserved.

\section{ABSTRACT}

The rate and extent to which an unchanged drug reaches the systemic circulation is called as bioavailability (BA). Bioavailability, a subcategory of absorption is one of the principal pharmacokinetic parameter determined for an active substance form a pharmaceutical product. It also indicates the fractional extent to which a dose of drug reaches its site of action or biological fluid from which the drug has access to its site of action. Physical properties of drug, drug formulation, route of administration, gastric emptying rate etc. are several factors affect the bioavailability of drug from its drug product. Poor solubility, enzymatic and transporters barrier, drug stability and short retention of the drug in stomach due to peristaltic movement are several factors decrease the bioavailability of the drug. This review deals with the bioavailability improvements techniques from poor permeation, lesser stability and short retention of the drug in stomach. Lipid based formulations; ion pairing and use of permeation enhancer are different methods to enhance the bioavailability through increase in permeation. Enteric coating, complexation and metabolism inhibitors lead to increase in drug stability. Bioadhesive polymers in formulation improve the gastro retention time serve as improved bioavailable product.

Key words: Bioavailability, Lipid based formulations, Enteric Coating, Gastro retentive Drug Delivery System.

\section{INTRODUCTION}

Bioavailability is a measurement of the rate and extent to which an unchanged drug reaches the systemic circulation. It is denoted by the letter f (Shargel L. 1999). It also indicates the fractional extent to which a dose of drug reaches its site of action or biological fluid from which the drug has access to its site of action. Figure 1, illustrates that a drug given orally must be absorbed initially from the stomach and intestine, but this may be limited by the characteristics of the dosage form and the drug's physicochemical properties. In addition, drug then passes through the liver, where metabolism and biliary excretion may occur before the drug enters the systemic circulation. Accordingly, a fraction of the administered and absorbed dose of drug will be inactivated or diverted before it can reach the general circulation and be distributed to its sites of action. If the metabolic or excretory capacity of the liver for the drug is large, bioavailability will be reduced substantially (the first-pass effect). This decrease in availability is a function of the anatomical site from which absorption takes place and the choice of the route of drug administration must be based on an understanding of these conditions (Goodman G. 2006).

\section{Necessity of Bioavailability Enhancement}

Enhancement of bioavailability is essential for many drugs like penicillin, atropine, chloramphenicol palmitate, griseofulvin etc., which have poor bioavailability due to

- Poor aqueous solubility and/or slow dissolution rate in biological fluids

- Poor stability of the dissolved drug at the physiologic $\mathrm{pH}$.

- Poor permeation through bio membrane due to inadequate partition coefficient.

\section{Approaches to overcome these problems}

\section{Pharmaceutics approach}

This involves modification of formulation, manufacturing process or the physicochemical properties of the drug without changing the chemical structure.

\section{Pharmacokinetic Approach}

Pharmacokinetics of the drug is altered by modifying its chemical structure. The approach of chemical structure modification has a number of hitches of being very expensive and time consuming. The requirement of repeated clinical studies and longer period for regulatory approval further makes the approach deplorable.

\section{Biological Approach}

Alteration in the route of drug administration such as changing from oral to parental route, also alter the bioavailability. The limitations of pharmacokinetic approaches to enhance bioavailability, the optimization of the formulation, manufacturing process or the physicochemical properties of the drug without changing the chemical structure are mainly aimed at enhancement of dissolution rate which lead to increase in bioavailability as it is the major rate limiting step in the absorption of most drugs (Brahmankar D. M, 2006). The Pharmaceutical approach for bioavailability enhancement can be applied by following techniques

- Enhancement of drug solubility and dissolution rate

- Enhancement of drug permeability

- Enhancement of drug stability

- Enhancement of gastrointestinal retention

Bioavailability enhancement through increase in drug permeability across biomembrane

\section{Lipid technologies}

Lipid-based formulations encompass a diverse group of formulations with very different physical appearance, ranging from simple triglyceride vehicles to more sophisticated formulations such as self-emulsifying drug delivery systems (SEDDS). Lipid-based drug delivery systems may contain a broad range of oils, surfactants and co-solvents. They represent one of the most popular approaches to overcome the absorption barriers and to improve the bioavailability of poorly water-soluble drugs. Oral lipid-based products entered the market in 1981 and presently having well commercially 
availability in oral formulations. Lipids open a wide area of different formulations for oral administration because they can be manufactured as solutions, suspensions, emulsions, self-emulsifying systems and micro-emulsions. Lipids offer the potential for enhancing drug absorption and oral bioavailability.

\section{Factors taken into consideration for lipid-based formulations \\ Lipid Digestion}

If the drug possesses high affinity to the lipid vehicle, it can be assumed that the API moves apparently together with vehicle in the GI-tract, revealing that digestibility of lipid would be as important as gastric emptying rate of the same. Thus, careful selection of the lipid vehicle can control the absorption rate of drug. GI lipid digestion consists of three sequential steps: (i) the dispersion of fat globules to yield a fine emulsion, (ii) the enzymatic hydrolysis of fatty acid esters at the emulsion-water interface and (iii) the desorption and dispersion of insoluble lipid products for subsequent absorption .

\section{Mean Emulsion Droplet Diameter}

The mean emulsion droplet diameter is a parameter indicating the quality of self-emulsifying formulations. The droplet size of SEDDS upon dilution with aqueous media is primarily influenced by the type and concentration of emulsifier. The higher the concentration of emulsifier, the smaller the emulsion droplet and the faster the drug release. Two techniques are commonly used to determine the mean emulsion droplet diameter: low angle laser light diffraction is applied for emulsions with droplet sizes $>1 \mu$ and quasielastic light scattering for investigations of submicron dispersions. In addition, the mean emulsion droplet diameter seems to be a very critical factor for prediction of the in-vivo performance of undigested lipid-based formulations, such as long chain triglycerides in case of Cyclosporine (Neoral ${ }^{\circledR}$ versus Sandimmune $\left.{ }^{\circledR}\right)$. Nevertheless, in case of predigested lipids such as medium chain monoglycerides or propylene glycol monoester of C8-C10 fatty acids, the mean emulsion droplet diameter may not be crucial in-vivo.

\section{Lipophilicity of Drug}

Highly hydrophobic drugs $(\log \mathrm{P}>6)$ can be taken up into the lymphatic system by partitioning into chylomicrons in the mesentery vein which has been demonstrated to be crucial for the absorption of the anti-malaria compound halofantrine. Furthermore, highly lipophilic retinoids are known to be transported in the intestinal lymph after oral administration.

\section{Type of Lipids}

The digestible lipids may influence absorption with differing method from that of non-digestible lipids. Commonly used digestible lipid vehicles are listed in Table 1. The lower the melting point of the fatty acid, the higher is the amount of drug absorbed.

\section{Drug Release}

Primarily, the characteristics of drug and lipid as well as their aqueous solubility are key factors to control drug release and absorption from lipid based formulations. Other issues to be considered are whether the drug is formulated in oil, SEDDS or emulsified form, the absorption pathway of the drug, the droplet size of the emulsion present in the intestine, the type of surfactants, the metabolic pathway of the lipids and the changes in gastric motility due to presence of lipids.

\section{Novel Lipid Formulations}

The carrier systems that have been most extensively studied to control the release of the incorporated substances are

- Lipid solutions \& suspensions

- Micro emulsions

- Self emulsifying drug delivery system (SEDDS)

- Solid lipid nanoparticles (SLN)

- Nanostructured lipid carriers (NLC)

- Lipid - drug conjugate (LDC)

- Liposomes (Basavaraj K 2011)

\section{Lipid Solutions \& Suspensions}

The simplest lipid-based formulations contain only one excipient such as oleic acid, $\alpha$-tocopherol, corn oil, peanut oil, sesame oil, medium chain triglycerides, or medium chain mono- and diglycerides. Many of the over-the-counter sold products contain polyethylene glycol or medium chain triglycerides as the solubilizing excipients. Some lipophilic drugs like steroids have good solubility in triacylglycerols. Table 2 illustrates some over the counter or some marketed formulations of Lipid Solutions. Therefore it is better to formulate a drug in an oily liquid and achieve good absorption which leads to enhanced bioavailability (Maulik Patel, 2011).

\section{Microemulsions}

The concept of microemulsion was introduced by Hoar and Schulman in 1943. They prepared first microemulsions by dispersing oil in an aqueous surfactant solution with addition of alcohol as a co-surfactant, leading to a transparent, stable formulation Some Patents of Microemulsions is shown in Table 3. The existence of this theoretical structure was later confirmed by use of various technologies. Thus as Shown in Figure 2 the microemulsions are defined as "a system of water, oil and amphiphilic compounds (surfactant and cosurfactant) which is a transparent, single optically isotropic, and thermodynamically stable liquid (Attwood D.1994) (Singh V. 2012).

\section{Self Emulsifying Drug Delivery System (SEDDS)}

The most popular approach for enhancing the bioavailability is the incorporation of the active lipophilic component into inert lipid vehicles, surfactant dispersions, self-emulsifying formulations with every formulation approach having its special advantages and limitations. Self-emulsifying drug delivery systems (SEDDS) are mixtures of oils and surfactants, ideally isotropic and sometimes containing cosolvents which emulsify spontaneously to produce fine oil-inwater emulsions when introduced into aqueous phase under gentle agitation. SEDDS have also been formulated using medium chain tri-glyceride oils and nonionic surfactants, the latter being less toxic. Some excipients and product formed by these excipients are listed in Table 5. Upon peroral administration, these systems form fine emulsions (or microemulsions) in gastro-intestinal tract (GIT) with mild agitation provided by gastric mobility. Some examples of Marketed formulations of SEDDS are Listed in Table 4 which have Potential advantages of enhanced oral bioavailability enabling reduction in dose, more consistent temporal profiles of drug absorption, selective targeting of drug(s) toward 
Vishal Sachdeva et al. Int. Res. J. Pharm. 2013, 4 (5)

specific absorption window in GIT and protection of drug(s)

from the hostile environment in gut.

Table 1: Vehicles Used For Lipid Formulations

\begin{tabular}{|c|c|}
\hline Class & Lipophilic vehicles \\
\hline Fatty acids & Oleic acid, Myristic acid, Caprylic acid, Capric acid \\
\hline Triglycerides of mediumchain fatty acids & Miglyol ${ }^{\circledR}$ 812, Captex ${ }^{\circledR}$ 355, Labrafac ${ }^{\circledR}$ \\
\hline Triglycerides of long-chain fatty acids & Soybean Oil, Peanut Oil, Corn Oil \\
\hline
\end{tabular}

Table 2: Marketed Lipid Solutions Formulations

\begin{tabular}{|c|c|c|c|}
\hline Brand Name & API & Manufacturer & Country \\
\hline Heminevrin & Clomethiazole & AstraZeneka & UK \\
\hline Marinol ${ }^{\circledR}$ & Dronabinol & Solvay Pharmaceuticals & USA \\
\hline Epadel ${ }^{\circledR}$ & Ethyl icosapentate & Mochida Pharmaceuticals & Japan \\
\hline
\end{tabular}

Table 3: Patents of Microemulsions

\begin{tabular}{|c|c|c|c|c|}
\hline Patent No. & Issue date & Original Assignee & Title & Reference \\
\hline US5023271 & Jun 11,1991 & California Biotechnology Inc & Pharmaceutical microemulsions & Vigne J.L 1991 \\
\hline US5055303 & Oct 8,1991 & KV Pharmaceutical Company & Solid controlled release Bioadherent Emulsions & Riley T.C et al, 1991 \\
\hline US5744155 & Apr 28, 1998 & $\begin{array}{l}\text { Friedman, Doron, Schwartz, } \\
\text { Joseph, Amselem, Shimon }\end{array}$ & $\begin{array}{c}\text { Bioadhesive emulsion preparations for enhanced drug } \\
\text { delivery }\end{array}$ & Friedman et al, 1998 \\
\hline US5925626 & Jul 20, 1999 & Fidia S.p.A. & $\begin{array}{c}\text { Hyaluronic acid fractions having pharmaceutical } \\
\text { activity, and pharmaceutical compositions containing } \\
\text { the same }\end{array}$ & Valle F.D et al, 1999 \\
\hline US6551605 & Apr 22, 2003 & Haarmann \& Reimer & $\begin{array}{c}\text { Diesters or polyesters of naphthalene dicarboxylic acid } \\
\text { as solubilizer/stabilizer for retinoids }\end{array}$ & Bonda C.A et al, 2003 \\
\hline US20020102280 & Jan. 08, 2002 & Anderson, David M. & Solvent systems for pharmaceutical agents & Anderson D.M, 2002 \\
\hline
\end{tabular}

\section{Table 4: Marketed Preparation of SEDDS}

\begin{tabular}{|c|c|c|}
\hline Name of product & Company name & Active drug \\
\hline VITA-SEDDS & Summit Vitamins USA & MULTI VITAMIN \\
\hline SEDDS VIT. D3 + Ca & Summit Vitamins USA & CALCIUM + VIT. D3 \\
\hline SEDDS CoQ10 & Summit Vitamins USA & CoQ10 \\
\hline SANDIMMUNE & Novartis USA & CYCLOSPORINE \\
\hline NORVIR & Abbott India Limited & RITONAVIR \\
\hline FORTOVASE & La Roche New Zealand & SAQUINAVIR \\
\hline \multicolumn{2}{|c}{ http://www.iherb.com/Summit-Vitamins }
\end{tabular}

Table 5: Surfactants, Co-Surfactant and Co-Solvent (Patel P. 2008)

\begin{tabular}{|l|l|}
\hline Excipients & Commercial products \\
\hline Surfactants/co-surfactants & \\
Polysorbate 20 (Tween 20) & Targretin soft gelatin capsule \\
Polysorbate 80 (Tween 80$)$ & Gengraf hard gelatin capsule \\
Sorbitan monooleate (Span 80) & Gengraf hard gelatin capsule \\
Polyoxy-35-castor oil(Cremophor RH40) & Gengraf hard gelatin capsule, \\
(Cremophor RH40) & Sandimmune soft gelatin capsules \\
Co-solvents & \\
Ethanol & Sandimmune soft gelatin Capsules \\
Glycerin & Nerol soft gelatin Capsule, \\
Polypylene glycol & Agenerage Oral solution, \\
Polyethylene glycol & Gengraf hard gelatin capsule \\
Lipid ingredients & \\
Corn oilmono,di,,tri-glycerides & Targretin soft gelatin capsule, \\
DL-alpha-Tocopherol & Agenerase soft capsule, \\
Fractionated triglyceride of coconut oil & Agenerase oral solution \\
(medium-chain triglyceride) & \\
Mixture of mono-and di-glycerides of caprylic/capric acid & Nerol soft gelatin Capsule, Nerol Oral \\
Medium chain mono-and di-glycerides & Solution \\
Corn oil & Rocaltrol soft gelatin capsule, \\
Olive oil & Avodat soft gelatin capsule \\
Oleic acid & Fortavase soft gelatin capsule \\
Sesame oil & Sandimmune soft gelatin capsule, \\
Hydrogenated soyabean oil & Depakene capsule \\
Hydrogenated vegetable oils & Sandimmune oral solution \\
Soyabean oil & Ritonavir soft gelatin capsule, \\
& Norvir soft gelatin capsule \\
\hline
\end{tabular}


Table 6: Patents of Enteric Coating Technology

\begin{tabular}{|c|c|c|c|c|}
\hline Patent No. & Issuing Date & Original Assignee & Rescription \\
\hline US4857337 & Aug 15, 1989 & $\begin{array}{c}\text { American Home Products Corp. } \\
\text { (Del) }\end{array}$ & Enteric coated aspirin tablets \\
\hline US5209933 & May 11, 1993 & Syntex (U.S.A.) Inc. & Long acting calcium channel blocker composition & Macfarlane et al, 1993 \\
\hline US5326586 & Jul 5, 1994 & BASF Aktiengesellschaft & Grabwoski et al, 1994 \\
\hline US5393333 & Feb 28, 1995 & $\begin{array}{c}\text { Societe Anonyme Societe } \\
\text { D'Exploitation De Produits Pour Les } \\
\text { Industries Chimiques S.E.P.P.I.C. }\end{array}$ & $\begin{array}{c}\text { Film-forming product for coating solid forms, process } \\
\text { for its manufacture and products coated with this film- } \\
\text { forming product }\end{array}$ \\
\hline US5643868 & Jul 1, 1997 & Autoimmune, Inc & $\begin{array}{c}\text { Method of treating or preventing type 1 diabetes by } \\
\text { oral administration of insulin }\end{array}$ & Weiner et al, 1997 \\
\hline US5733575 & Mar 31, 1998 & BPSI Holdings, Inc & $\begin{array}{c}\text { Enteric film coating compositions, method of coating } \\
\text { therewith, and coated forms }\end{array}$ & Mehra D.K et al, 1998 \\
\hline US5877309 & Mar 2, 1999 & ISIS Pharmaceuticals, Inc. & Antisense oligonucleotides against JNK & McKay R. 1999 \\
\hline US7122207 & Oct 17, 2006 & Bristol-Myers Squibb Company & High drug load acid labile pharmaceutical composition & Ullah et al, 2006 \\
\hline
\end{tabular}

Table 7: Patents of FDDS (Rabadia N. 2012)

\begin{tabular}{|c|c|c|c|}
\hline Drug & Dosage form & Patent no. & Reference \\
\hline Valsartan & Swelling tablet & WO PCT 2008027945 & Javant N. et al, 2008 \\
\hline Theophylline & Multi layered tablet & US PATENT 5783212 & Fassihi R. et al 1998 \\
\hline Amoxicillin & SR floating capsule & US PATENT 2006121106 & Kerc J. et al, 2006 \\
\hline Cimetidine, Ranitidine & Powder, tablet & US PATENT 5288506 & Spickett R. G et al, 1994 \\
\hline Methotrexate & SR tablet & US PATENT 2008268045 & Dervieux T et al, 2008 \\
\hline
\end{tabular}

Table 8: Marketed preparations of FDDS (Shruti s. 2011)

\begin{tabular}{|c|c|c|c|}
\hline Brand name & Delivery & Active drug \\
\hline Madopar HBS & Floating capsule & Benserazide (25mg) and L-Dopa (100mg) & Roche Products, USA \\
\hline Topalkan $\mathbb{}$ & Floating liquid alginate preparation & Al - Mg antacid & Pierre Fabre Drug, France \\
\hline Cifran OD $\AA$ & Gas-generating floating form & Ferrous sulphate & Ranbaxy, India \\
\hline Conviron ${ }^{\circledR}$ & Colloidal gel forming FDDS & Ranbaxy, India \\
\hline Liquid Gaviscon ${ }^{\circledR}$ & Effervescent Floating liquid alginate preparations & Al hydroxide (95 mg), Mg Carbonate (358 mg) & GlaxoSmithkline, India \\
\hline
\end{tabular}

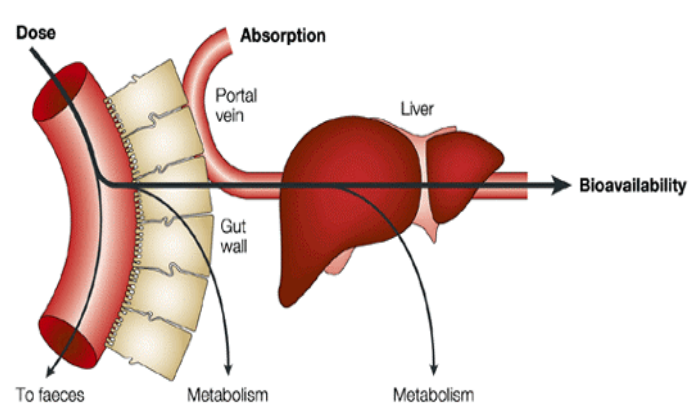

Figure 1: Schematic Representation of Bioavailability

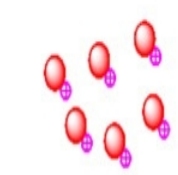

Water-soluble cationic drug (Mitoxantrone hydrochlonde, MTO)

Counter-ionic polymer

(Dextran sulfate sodium, DS)

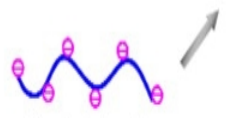

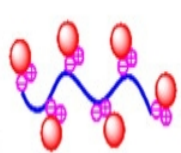

\section{MTO.DS} electrostatic complex

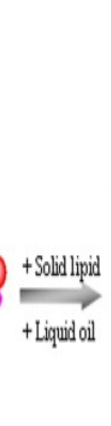
MTO-loaded nanostructured lipid.DS hybond camers (MTO-NLDCs)

Figure 3: NLC of Mitoxantrone Hydrochloride

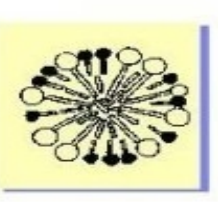

Microemulsion (10-200 nm)

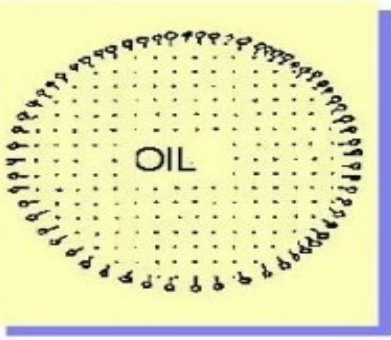

Emulsion

$(>1000 \mathrm{~nm})$
Figure 2: Particle Size of Emulsion \& Microemulsion

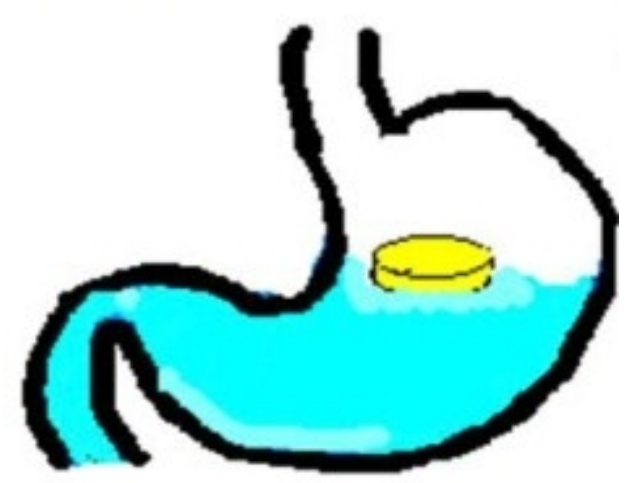

Figure 4: Representation of Floating Drug Delivery System 
Merits of SEDDS

Selective targeting of drug(s) toward specific absorption window in GIT, Protection of $\operatorname{drug}(\mathrm{s})$ from the hostile environment in gut, Enhanced oral bioavailability enabling reduction in dose, More consistent temporal profiles of drug absorption, Control of delivery profiles, Reduced variability including food effects.

\section{Research Materialized in SEDDS}

- Gattefosse Corporation, France, has patented SEDDS formulation of Indomethacin which offered two fold increase in bioavailability in rats compared to conventional formulations (Crison J. et al, 1999)

- Charman et.al reported improvement in pharmacokinetic parameters of Halofantrine (highly lipophillic antimalarial drug) and reduction in inter-subject variability when administered as SEDDS (Charman et al, 1998)

- K Itoh observed improvement in biopharmaceutical properties of N-4472, an investigational lipophillic drug having lipolytic activity in SEDDS formulation. (Itoh K. et al, 2003)

- Nazzal et al observed improvement in pharmacokinetics and two-fold increase in bioavailability of Ubiquinone, a lipophillic drug used as an anti-anginal agent and as antioxidant when given as SEDDS (Gupta N. 2009)

\section{Solid-Lipid Nanoparticle}

Solid lipid nanoparticles (SLN) represent an alternative carrier system to tradition colloidal carriers such as emulsions, liposomes and polymeric micro and nanoparticles. This system was introduced in 1991. Nanoparticles made from solid lipids are attracting major attention as novel colloidal drug carrier for intravenous applications as they have been proposed as an alternative particulate carrier system. SLN are sub-micron colloidal carriers ranging from 50 to $1000 \mathrm{~nm}$, which are composed of physiological lipid, dispersed in water or in aqueous surfactant solution. SLN offer unique properties such as small size, large surface area, high drug loading and the interaction of phases at the interface and are attractive for their potential to improve performance of pharmaceuticals (Ekambaram P.2012). In order to overcome the disadvantages associated with the liquid state of the oil droplets, the liquid lipid was replaced by a solid lipid, which eventually transformed into solid lipid nanoparticles. Even due to the several reasons viz Lipids enhance oral bioavailability and reduce plasma profile variability, Better characterization of lipoid excipients \& An improved ability to address the key issues of technology transfer and manufacture scale-up, lead to the increased interest in lipid based drug delivery system.

\section{Merits of SLN}

Small size and relatively narrow size distribution which provide biological opportunities for site-specific drug delivery by SLNs. SLNs shows controlled release of active drug over a long period and can be sterilized by autoclaving or gamma irradiation, ability to be lyophilized as well as spray dried, easy to produce in industrial scale by hot dispersion method, no toxic metabolite and economical as compared to other dosage forms (Sailaja K. 2011).

\section{Research Materialized in SLNS}

- SLNs have been reported to be useful as drug carriers to treat neoplasms. Tumour targeting has been achieved with
SLNs loaded with drugs like methotrexate and camptothecin. Tamoxifen an anticancer drug is incorporated in SLN to prolong release of drug after i.v. administration.

- Mitoxantrone-loaded SLN local injections were formulated to reduce the toxicity and improve the safety and bioavailability of drug41. Efficacy of doxorubicin (Dox) has been reported to be enhanced by incorporation in SLNs. In the methodology the Dox was complex with soybean-oil-based anionic polymer and dispersed together with a lipid in water to form Dox-loaded solid lipid nanoparticles. The system has enhanced its efficacy and reduced breast cancer cells (Vishvajit A. 2010).

\section{Nanostructured Lipid Carriers}

The application of NLC as a drug delivery system is enhanced by eliminating the use of organic solvents in the preparation stage and using the hot high-pressure homogenization technique an example of NLC of mitoxantrone hydrochloride is shown in Figure 3. Polysorbate 20 and polysorbate 80 are non-ionic surfactants commonly used as excipients and emulsifiers in medications for parenteral administration. However, their efficiency in the stabilization of NLC is yet to be elucidated.

\section{Research Materialized in NLC}

- Souto and Müller, 2006 reported that among the nanostructured lipid carriers that contain solid lipids together with liquid oils are $\operatorname{Miglyol}{ }^{\circledR}, \alpha$-tocopherol etc. (Muller et al. 2006)

- Müller et al. 2002 reported that the presence of liquid lipids with different fatty acid C-chains produces NLC with less organized crystalline structure and therefore provides better loading capacity for drug accommodation. Liquid lipids are better solubilizers of drugs than solid lipids. (Müller et al. 2002)

\section{Lipid - Drug Conjugate (LDC)}

The major problem of SLN is the low capacity to load hydrophilic drug due to partitioning effect during the production process. Only highly potent low dose hydrophilic drug may be suitably incorporated in the solid lipid matrix, in order to overcome this problem, the LDC nanoparticles with drug loading capacity up to $33 \%$ have been developed. An insoluble drug- lipid conjugate bulk is first prepared either by salt formation or by covalent linking. This LDC is further processed with an aqueous surfactant solution such as tween to a nanoparticle formulation, using high pressure homogenization. Such matrices may have potential application in brain targeting of hydrophilic drug in serious protozoal infections. Increase in bioavailability is achieved by LDC with the advantage of control \& targeted drug release. LDCs are easy to validate, scale up \& sterilize (Patidar A. 2010).

\section{Ion Pairing}

Ion-pair, a single unit, is a pair of oppositely charged ions held together by Coulomb attraction without formation of a covalent bond. Hydrophobic ion pairing technique has been used to increase the hydrophobicity of molecules containing ionizable groups by stoichiometric replacement of the polar counter ions with more hydrophobic ones. Ion-paired delivery has emerged as a viable approach for enhancing solubility of ionic molecules in non-polar solvents, augmentation of protein and DNA transport and addressing 
the poor bioavailability issues of hydrophilic drugs. Ionpairing can be a valuable tool for enhancing solubility and stability in an organic solvent. The complexes formed can be formulated into particulates with higher drug loading. This technique has found unique opportunities in delivery of polyelectrolytes (e.g. peptide, proteins and polynucleotide) by using them as hydrophobic ion-paired moieties for designing particulates and microemulsions that during formulation require direct solubilization in organic solvents. Additionally, this approach can also improve stability and permeability across biological membranes. Formation of ion pairs normally does not entail an alteration in the structure and function of drug. Thus, this approach can assist in designing better dosage forms for alternative routes of administration (Preeti K. 2011).

\section{Research Materialized in Ion Pairing}

- Sarveiya et al. (2004) reported a 16-fold increase in the steady-state flux of ibuprofen ion-pairs across a lipophilic membrane. They determined the influence of $\mathrm{pH}$ and ionpairing on the permeation of ibuprofen across polydimethylsiloxane (PDMS) membrane. Diffusion studies at different $\mathrm{pH}$ values $(4.0,5.0,6.0,7.0$ and 8.0) indicated that ibuprofen sodium flux increased significantly with increasing $\mathrm{pH}$ from 4.0 to 7.0. Above $\mathrm{pH}$ 7.0, a decrease in diffusion was observed. The permeability coefficient increased with an increase in the amount of unionized acid.(Sarveiya et al.2004)

- Sineerat et al. (2008) investigated a water insoluble complex of cationic propranolol $\mathrm{HCl}$ with anionic sodium lauryl sulfate. They concluded that the 'propranololsodium lauryl sulfate complex' provided promising sustained drug delivery and were feasible to be encapsulated in microparticles for a more sustained drug release effect. (Sineerat et al.2008)

\section{Penetration Enhancers}

Peroral delivery of hydrophilic drugs is one of the greatest challenges in biopharmaceutical research. Hydrophilic drugs usually present low bioavailability after oral administration. One of the causes of this low bioavailability is their poor intestinal permeation through the paracellular pathway. This pathway is actually restricted by the presence of tight junctions at the apical side of the enterocytes. In the last few years, great interest has been focused on the structure and cellular regulation of tight junctions, materializing in more in-depth knowledge of this intestinal barrier. Regular efforts are being made to develop agents that can modulate tight junctions and magnify the paracellular permeability of hydrophilic compounds without causing significant intestinal damage. These compounds are called as penetration enhancers or promoters. Based on the research conducted in the last decade it has become clear that several sodium salts of medium chain fatty acids [caprylate $\mathrm{C} 8(\mathrm{CH} 3-(\mathrm{CH} 2) 6-$ $\mathrm{COOH})$, caprate $\mathrm{C} 10(\mathrm{CH} 3-(\mathrm{CH} 2) 8-\mathrm{COOH})$ and laurate $\mathrm{C} 12$ (CH3-(CH2)10-COOH)] are able to enhance the paracellular permeability of hydrophilic compounds (Jose M. 2005).

\section{Bioavailability enhancement through increased drug stability \\ Enteric coating}

Oral site-specific drug delivery systems are widely used for the treatment of a variety of bowel diseases along with the improved systemic absorption of drugs, which are unstable in the stomach. However, the micro-environment in the gastrointestinal tract and varying absorption mechanisms usually creates barrier for the formulation development and optimization of oral drug delivery. An enteric coating on a solid dosage form can be used for delivery of therapeutic agent into the intestinal region. Such systems are designed to provide protection to tablets in the stomach. A thick coat on solid dosage form causes a delay in the drug release in the small intestine and slows down drug release, which is both $\mathrm{pH}$ and time-controlled. This ensures drug delivery to be colon specific. For the preparation of such tailor-made formulations, the selection of a polymer with a suitable coat level is the major factor to be considered. Most of the commercially available systems for colon specific drug delivery utilize Eudragit (L-100/ S-100) or cellulose acetate phthalate (CAP), shellac (SH) and ethyl cellulose (EC). Eudragit S-100 (ES) is a methacrylic acid methyl methacrylate co-polymer, which is soluble at a $\mathrm{pH}$ of 7. CAP is also an effective enteric coating material as it dissolves at a $\mathrm{pH}$ of 6 . It is used at a concentration of $0.5-0.9 \%$. Patents of Enteric Coating Technology is Enlisted in Table 6. (Punia S. 2012).

\section{Complexation}

Complexation of drug can be used to increase the drug stability in gastro intestinal tract (GIT). This objective can be served predominantly with Cyclodextrin. The central cavity of the cyclodextrin molecule is lined with skeletal carbons and ethereal oxygen's of the glucose residues. It is therefore lipophilic. The polarity of the cavity has been estimated to be similar to that of aqueous ethanolic solution. It provides a lipophilic microenvironment into which suitably sized drug molecules may enter and be included. No covalent bonds are formed or broken during drug-cyclodextrin complex formation, and in aqueous solutions, the complexes are readily dissociated. Free drug molecules are in equilibrium with the molecules bound within the cyclodextrin cavity. Measurements of stability or equilibrium constants $(K \mathrm{c})$ or the dissociation constants $(K d)$ of the drug-cyclodextrin complexes are important since this is an index of changes in physicochemical properties of a compound. (Thorsteinn L. 1996)

\section{Metabolism Inhibitors}

Administration of a drug with its metabolism inhibitor, results in increase fractional absorption \& higher bioavailability. This approach seems to be promising approach to overcome enzymatic barriers of drugs such as peptides \& proteins. Important example of a metabolic inhibitor is grapefruit juice. This can produce several clinically significant drug interactions through enzyme inhibition. These include a reduction in the metabolism of cyclosporin, calcium channel blockers and simvastatin.

\section{Bioavailability Enhancement through Gastrointestinal Retention}

Gastro-retentive drug delivery systems (GRDDS) are designed on the basis of delayed gastric emptying and are intended to localize the drug in stomach or within the upper part of small intestine until the entire is released. Excipients that are bioadhesive; swell on hydration can promote gastric retention and ultimately absorption. 
Types of GRDDS

\section{Floating drug delivery system (FDDS)}

Floating systems or hydrodynamically controlled systems as shown in Figure 4 are low-density systems that have sufficient buoyancy to float over the gastric contents and remain buoyant in the stomach without affecting the gastric emptying rate for a prolonged period of time. While the system is floating on the gastric contents, the drug is released slowly at the desired rate from the system. After release of drug, the residual system is emptied from the stomach. This system lead to increased gastric retention time (GRT) and control of the fluctuations in plasma drug concentration. A minimal level of floating force $(\mathrm{F})$ is also required to keep the dosage form reliably buoyant on the surface of the meal. A minimal gastric content is also required to allow the proper achievement of the buoyancy retention. Many buoyant systems have been developed based on granules, powders, capsules, tablets, laminated films and hollow microspheres which are listed in Table 8 along with some Patent in FDDS which are listed in Table 7. (Mayavanshi A. 2008). Cook JD et al, 1990 reported that the bioavailability of riboflavin controlled release-gastro retentive dosage form (CR-GRDF) is significantly enhanced in comparison to the administration of non-GRDF CR polymeric (Goyal M. 2011).

\section{Bioadhesive Drug Delivery System}

The relatively short gastric emptying time in humans, which normally averages 2-3 hrs through the major absorption zone (stomach or upper part of the intestine), can result in incomplete drug release from the drug delivery system leading to diminished efficiency of the administered dose. Localization of a drug delivery system in a specific region of the GIT offers numerous advantages, especially for drugs having narrow absorption window. The intimate contact of the dosage form with the absorbing membrane has the potential to maximize drug absorption and may also influence the rate of drug absorption. These considerations have lead to the development of oral sustained release dosage forms possessing gastric retention potential. The primary concern in the development of once daily oral sustained release dosage form is not just to prolong the delivery of drugs for $24 \mathrm{hrs}$ but also to prolong the presence of dosage forms in the stomach or somewhere in the upper small intestine. Gastroretentive dosage forms through local drug release will greatly enhance the pharmacotherapy of the stomach leading to high drug concentrations at the gastric mucosa, which are sustained over a long period of time. Gastroretentive delivery system leads to Improvement of bioavailability and therapeutic efficacy of the drugs and possible reduction of dose e.g. Furosemide, Maintenance of constant therapeutic levels over a prolonged period and thus reduction in fluctuation in therapeutic levels minimizing the risk of resistance especially in case of antibiotics. e.g. Beta-lactam antibiotics (penicillin's and cephalosporin's), Retention of drug delivery systems in the stomach, prolongs overall gastrointestinal transit time thereby increasing bioavailability of sustained release delivery systems intended for once-a-day administration. e.g. Ofloxacin (Zate S.U, 2011).

\section{CONCLUSION}

Poor solubility, enzymatic and transporters barrier, drug stability and short retention of the drug in stomach due to peristaltic movement are several factors decrease the bioavailability of the drug. This Problem can be overcome by using Lipid based formulations, ion pairing and use of permeation enhancer, to enhance the bioavailability through increase in permeation. Enteric coating, complexation and metabolism inhibitors lead to increase in drug stability. Bioadhesive polymers in formulation improve the gastro retention time serve as improved bioavailable product.

\section{REFERENCES}

1. Shargel L \& WU- Pong S et al. Applied Biopharmaceutics \& Pharmacokinetics - Sixth Edition the McGraw-Hill Companies New York; 2005.p. 3

2. Brunton LL. Goodman \& Gilman's the Pharmacological Basis of Therapeutics $-11^{\text {th }}$ ed. Mcgraw-hill Medical Publishing Division, New York; 2006. p. 2-3

3. Brahmankar DM \& Jaiswal SB. Biopharmaceutics \& Pharmacokinetics: A treatise, $1^{\text {st }}$ ed, Vallabh Prakashan; 2006.p. 345-347

4. Basavaraj KN et al. Functions of Lipids for Enhancement of Oral Bioavailability of Poorly Water-Soluble Drugs Scientia Pharmaceutica 2011;79:705-727.http://dx.doi.org/10.3797/scipharm.1105-09 PMid:22145101 PMCid:3221495

5. Patel $\mathrm{M}$ et al. A Review: novel oral based formulation for poorly soluble drugs, international journal of pharmaceutical science \& nanotechnology. 2011; 3(4): 1182-92.

6. Attwood D. Microemulsions in Colloidal drug delivery systems (J. Kreuter ed.), Marcel Dekker, New York; 1994.p. 650

7. Singh V et al. Recent advances in pharmaceutical emulsion technology. Journal of Pharmacy Research. 2012; 5(8): 4250-4258.

8. Vigne et al. Pharmaceutical Microemulsion, United States Patent; 1991.p. 5023271

9. Riley TC et al. Solid controlled release Bioadherent Emulsions, United States Patent; 1991.p. 5055303

10. Friedman et al. Bioadhesive emulsion preparations for enhanced drug delivery, United States Patent; 1998.p. 5744155

11. Valle FD et al. Hyaluronic acid fractions having pharmaceutical activity, and pharmaceutical compositions containing the same, United States Patent; 1999.p. 5925626

12. Bonda CA et al. Diesters or polyesters of naphthalene dicarboxylic acid as solubilizer/stabilizer for retinoids, United States Patent; 2003.p. 6551605

13. Anderson DM. Solvent systems for pharmaceutical agents, United States Patent; 2002.p. 20020102280

14. Patel $P$ et al. Self Emulsifying Drug Delivery System: A Review Research J. Pharm. and Tech. 2008; 1(4): 313.

15. Crison $\mathrm{J}$ et al. Method \& Formulation for increasing Bioavailability, United States Patent; 1999.p. 5993858

16. Charman et al. Formulation design and bioavailability assessment of lipidic self-emulsifying formulations of halofantrine, International Journal of Pharmaceutics 1998; 167: 155-164. http://dx.doi.org/ 10.1016/S0378-5173(98)00054-4

17. Itoh $\mathrm{K}$ et al. Improvement of physicochemical properties of N-4472. Part III. VC/N-4472 complex formation and self-association in aqueous solution, Chemical \& Pharmaceutical Bulletin. 2003; 51(1): 40-5. http:/ /dx.doi.org/10.1248/cpb.51.40

18. Gupta $\mathrm{N}$ et al. Enhancement of oral bioavailability of lipophillic drugs from self microemulsifying drug delivery system (SMEDDS), International Journal of Drug Delivery \& Research, 2009; 1(1): 10-18.

19. Ekambaram P et al. Solid Lipid Nanoparticles: A Review, scientific review \& chemical communication 2012; 2(1): 80-102 http:// www.iherb.com/Summit-Vitamins

20. Sailaja K. Formulation of solid lipid nanoparticles and their applications, Current Pharma Research CPR 2011; 1(2): 197-203

21. Kamble VA et al. Solid lipid Nanoparticles as drug delivery system, international Journal of Pharma and Bio Sciences 2010; 1(3): 201-205.

22. Chee Wun How et al. Physiochemical properties of Nanostructured lipid carrier, African Journal of Biotechnology 2011; 10(9): 1684-1689.

23. Muller et al, Lipid nanoparticles (SLN, NLC) in cosmetic and pharmaceutical dermal products, International Journal of Pharmaceutics, 2006; 366(1-2): 170-184.

24. Patidar A et al. A review on novel lipid based Nanocarier, International Journal of Pharmacy and Pharmaceutical Sciences 2010; 2(4): 30-35.

25. Preeti KS and Swarnali DP. Ion-paired Drug Delivery: An Avenue for Bioavailability Improvement, Sierra Leone Journal of Biomedical Research 2011; 3(2): 70-76.

26. Sarveiya et al. Ion-pairs of ibuprofen: increased membrane diffusion, Journal of Pharmacy and Pharmacology, 2004; 56(6): 717-724. http: //dx.doi.org/10.1211/0022357023448 PMid:15231036

27. María $\mathbf{J}$ et al. Intestinal Absorption Enhancement via the Paracellular Route by Fatty Acids, Chitosans and Others: A Target for Drug Delivery, Current Drug Delivery; 2002. p. 9-22

28. Punia $\mathrm{S}$ et al. Enteric coating technology - an overview Novel Science, International Journal of Pharmaceutical Science. 2012; 1(7): 435-438. 
29. Miller et al. Enteric coated aspirin tablets, United States Patent; 1989.p. 4857337

30. Macfarlane et al. Long acting calcium channel blocker composition, United States Patent; 1993.p. 5209933

31. Grabwoski et al. Coating of drug forms, United States Patent; 1994.p. 5326586

32. Trouve et al. Film-forming product for coating solid forms, process for its manufacture and products coated with this film-forming product, United States Patent; 1995.p. 5393333

33. Weiner et al. Method of treating or preventing type 1 diabetes by oral administration of insulin United States Patent; 1997.p. 5643868

34. Mehra DK et al. Enteric film coating compositions, method of coating therewith, and coated forms, United States Patent; 1998.p. 5733575

35. McKay R. Antisense oligonucleotides against JNK, United States Patent; 1999.p. 5877309

36. Ullah et al. High drug load acid labile pharmaceutical composition, United States Patent; 2006.p. 7122207

37. Thorsteinn L and Marcus E. Pharmaceutical applications of cyclodextrins. Drug solubilization and stabilization, journal of pharmaceutical science 1996; 85: 10.

38. Goyal $\mathrm{M}$ et al. Floating Drug Delievery System, Journal of Current Pharmaceutical research, 2011;05(1): 7-18.
39. Rabadia N. The Floating Drug Delivery System \& Its Impact On Calcium Channel Blocker International journal of Pharmaceutical Research \& Development. 2011; 3(12): 107-131

40. Javant $\mathrm{N}$ et al. World Intellectual Property Organisation Patent; 2008.p. 2008027945

41. Fassihi R et al. Multi layered tablet, United States Patent; 1998.p. 5783212

42. Kerc J et al. SR floating capsule of Amoxicillin, United States Patent; 2006.p. 2006121106

43. Spickett RG et al. Antacid with Prolonged Gastric Residence Time, United States Patent; 1994.p. 5288506

44. Dervieux T et al. Sustain Release Methotrexate Formulation, United States Patent; 2008.p. 2008268045

45. Shruti SA. Review On: Recent Advancement of Stomach Specific Floating Drug Delivery System "International Journal of Pharmaceutical \& Biological Archives 2011; 2(6): 1561-1568.

46. SU Zate et al. Gastro Retentive Bioadhesive Drug Delivery System: A Review ,International Journal of Pharm Tech Research 2010; 2(2): 1227-1235.

Cite this article as:

Vishal Sachdeva, Mahesh Kumar Kataria. Enhancement of bioavailability through increase in drug permeation, stability and retention time. Int. Res. J. Pharm. 2013; 4(5):59-66

Source of support: Nil, Conflict of interest: None Declared 